\title{
Qualidade vida, sobrecarga, suporte social, ajustamento conjugal e morbidade psicológica em cuidadores de idosos com dependência funcional
}

\author{
Maria da Graça Pereira \\ Helena Carvalho \\ Universidade do Minho - Braga, Portugal
}

\begin{abstract}
Resumo
Mudanças recentes ao nível dos serviços de saúde resultaram em internamentos mais curtos nos hospitais e na procura de substitutos para cuidar dos doentes, levando a que as famílias assumissem frequentemente esses cuidados. Os familiares que se tornam cuidadores têm que lidar com várias mudanças nas suas vidas, especialmente quando o familiar de quem cuidam se encontra dependente. Este estudo focou-se nas implicações do cuidar ao nível da sobrecarga, atividadades de vida diária, qualidade de vida, satisfação com o suporte social, ajustamento da díada, depressão e ansiedade. Participaram neste estudo 109 cuidadores informais de idosos funcionalmente dependentes. Os instrumentos usados foram: Índice de Barthel; Escala de Sobrecarga do Cuidador; WHOQOL-BREF; Escala da Satisfação com o Suporte Social; Escala de Ajustamento Conjugal Revista; Inventário de Beck para a Depressão e o Inventário de Avaliação da Ansiedade Estado e Traço. Os resultados revelaram que a qualidade de vida estava negativamente relacionada com a sobrecarga e morbidade e positivamente com a satisfação com os amigos. A sobrecarga e qualidade de vida correlacionaram-se com o suporte social total. O ajustamento conjugal moderou a relação entre depressão e qualidade de vida (dimensões física e psicológica), mas não entre sobrecarga e qualidade de vida. Os cuidadores cônjuges apresentam menor qualidade de vida e os cuidadores filhos, mais morbidade psicológica. Verificou-se também que cuidadores que possuem apoio ao nível da prestação de cuidados apresentam maior satisfação conjugal. Os resultados enfatizam a importância de intervir junto desta população no sentido de aumentar a qualidade de vida dos cuidadores.
\end{abstract}

Palavras-chave: Cuidadores, Idosos, Qualidade de vida, Sobrecarga, Morbidade.

\section{Quality of life, burden, social support, marital adjustment and psychological morbidity in caregivers of elders with functional dependence}

\begin{abstract}
Changes in medical practice resulted in shorter hospitalization and in the search for substitute caregivers leading families to assume care. Family members who become caregivers need to deal with changes in their lives on several levels, particularly when caregiving falls upon a dependent family member. This study focused on the implications of caregiving on burden, life activities, quality of life, satisfaction with social support, dyadic adjustment, depression and anxiety. 109 informal caregivers of functionally dependent elders participated in the study. The instruments used were: Barthel Index, Zarit Burden Interview, WHOQOL-BREF, Satisfaction with Social Support Scale, Revised Dyadic Adjustment Scale, Beck Depression Inventory and State Trait Anxiety Inventory. The results revealed that quality of life was inversely related with burden and psychological morbidity and positively with satisfaction with friends. Burden and quality of life were correlated with social support. In turn, marital adjustment was found to be a moderator in the relationship between depression and quality of life (physical and psychological dimensions) but not between burden and quality of life. Caregivers that were partners of those they cared for showed lower quality of life and adult children caregivers
\end{abstract}

Endereço para correspondência: Universidade do Minho, Escola de Psicologia, Campus de Gualtar , 4710-057. Braga, Portugal. E-mails: gracep@psi.uminho.pt / enfermeirahelena@gmail.com.

Artigo aceito durante a gestão do editor Gerson Tomanari. 
revealed more psychological morbidity. Caregivers who received support in caregiving tasks showed more marital satisfaction. Results emphasize the importance of intervention with this population particularly regarding psychological morbidity and burden, in order to increase caregivers' quality of life.

Keywords: Caregivers, Elders, Quality of life, Burden, Morbidity.

\section{Calidad de vida, sobrecarga, apoyo social, ajuste marital y morbilidad psicológica en los cuidadores de ancianos con dependencia funcional}

\section{Resumen}

Los recientes cambios a nivel de los servicios de salud dieron lugar a hospitalizaciones más cortas y a la búsqueda de sustitutos para el cuidado de los pacientes, haciendo con que las familias asumieran frecuentemente esos cuidados. Miembros de la familia que se convierten en cuidadores tienen que lidiar con varios cambios en sus vidas, especialmente, cuando el familiar depende de su atención. Este estudio se centró en las implicaciones de la tensión a nivel de sobrecarga, actividades de la vida diaria, la calidad de vida, satisfacción con el apoyo social, depresión y ansiedad. En este estudio participaron 109 cuidadores informales de ancianos con dependencia funcional. Los instrumentos utilizados fueron el Î́ndice de Barthel, la Escala de sobrecarga del cuidador, WHOQOL-BREF, Escala de Satisfacción con el Apoyo Social, la Escala de Ajuste Marital Revisado, Inventario de Depresión de Beck y la evaluación del Estado y Rasgo de ansiedad. Los resultados revelaron que la calidad de vida estaba relacionada negativamente con la sobrecarga y morbilidad, y positivamente con la satisfacción con los amigos. La sobrecarga y la calidad de vida se correlacionaron con el apoyo social total. El ajuste marital moderó la relación entre la depresión y la calidad de vida (las dimensiones físicas y psicológicas), pero no entre la sobrecarga y la calidad de vida. Los cónyuges cuidadores tienen menor calidad de vida y los cuidadores hijos más morbilidad psicológica. También se constató que los cuidadores que tienen soporte a nivel de la prestación de cuidados tienen una mayor satisfacción conyugal. Los resultados enfatizan la importancia de intervenir en esta población para aumentar la calidad de vida de los cuidadores.

Palabras clave: Cuidadores, Personas mayores, Calidad de vida, Sobrecarga, Morbilidad.

\section{Introdução}

As famílias são reconhecidas como o principal contexto para a promoção e manutenção da autonomia e saúde dos seus membros e como a principal entidade prestadora de cuidados em situações de dependência dos seus familiares (Mendes, 2004; Richards \& Lilly, 2001). O termo cuidador informal refere-se à pessoa que proporciona a maior parte do cuidado ao idoso (Aleixo, Augusto \& Gomes, 2002; Lage, 2005). Cuidador informal e família cuidadora são termos que se reportam a indivíduos não pagos, tais como família, amigos e vizinhos que prestam cuidados, podendo trabalhar ou não a tempo inteiro e viver junto ou separadamente da pessoa cuidada (Jamura, 1997; Lage, 2004).

O cônjuge, geralmente, encontra-se na primeira linha da prestação de cuidados. Se este não estiver disponível ou capaz de prestar cuidados, são os filhos que habitualmente assumem esse papel, partilhando-o com o seu parceiro(a). Wolff e Kasper (2006) verificaram que $41,3 \%$ dos cuidadores de idosos dependentes são filhos adultos e $38,4 \%$ são cônjuges. Cônjuges cuidadores relatam mais sintomas de depressão, maior sobrecarga financeira e física e menor qualidade de vida psicológica (McPherson, Wilson, Chyurlia, \& Leclerc, 2010). As repercussões de uma dependência interferem com diversos domínios da qualidade de vida (QV) do idoso e família (Martins, 2004). As limitações da capacidade funcional do idoso comprometem a sua $\mathrm{QV}$ bem como a QV do cuidador, já que frequentemente os cuidadores dos idosos dependentes passam por situações de grande sobrecarga física, psicológica e emocional, com 
escassos apoios quer sociais e/ou familiares (Zarit, Reever \& Bach-Peterson, 1983).

De uma forma geral, os cuidadores apresentam morbidade psicológica superior aos indivíduos da mesma idade não cuidadores (Sequeira, 2007). Verifica-se que 53,3\% dos cuidadores informais apresentavam depressão clínica e 62,2\%, ansiedade. A ansiedade e a depressão apresentaram fortes relações com os níveis de sobrecarga (Parks \& Pilisuk, 1991). Em termos de severidade de depressão, Martín (2005) verificou que $24 \%$ dos cuidadores da sua amostra apresentavam níveis leves de depressão, 6\% níveis de depressão moderada e $3 \%$ níveis de depressão grave. Assim, mais de metade dos cuidadores apresentavam níveis preocupantes de ansiedade e depressão que interferiam no seu bem-estar psicológico. Por sua vez, a morbidade psicológica encontra-se ainda relacionada com a exigência de cuidados, não se verificando diferenças significativas nos cuidadores, quer sejam cônjuges, filhos ou noras, a este nível (Pinquart \& Sorensen, 2011).

No que concerne à qualidade de vida do cuidador, a literatura indica que os cuidadores apresentam com muita frequência cansaço físico e a percepção que a sua saúde, no global, decaiu desde que a prestação de cuidados do idoso dependente foi assumida (Imaginário, 2003; Salgueiro, 2008). Além disso, os cuidadores referem fadiga geral, dores nas costas, esgotamento físico e mental, diminuição da força e resistência, nervosismo, irritabilidade, ansiedade, insônias e humor depressivo (Brito, 2002). Martins (2005) elaborou um estudo sobre a qualidade de vida e bem-estar dos doentes e familiares cuidadores e concluiu que quanto menor a sobrecarga física, emocional e social, menor a morbidade psicológica do cuidador e, consequentemente melhor a sua qualidade de vida.

Dentre as consequências negativas que correspondem a alterações ou mudanças específicas na vida dos cuidadores informais, a sobrecarga é sem dúvida importante na literatura gerontológica (Lage, 2004; Paúl, 2005; Pearlin, Mullan, Semple \& Skaff, 1990). $\mathrm{O}$ termo sobrecarga é reportado às consequências físicas, psicológicas e sociais resultantes do ato de cuidar de um indivíduo quando este se encontra dependente da prestação ininterrupta de cuidados (Pearlin et al., 1990). De fato, cuidar de um familiar idoso representa uma situação de stress, uma ameaça ao equilíbrio do funcionamento normal do indivíduo, sendo um compromisso que compete com o desenvolvimento das atividades do cuidador (Williamson \& Schulz, 1990). A fase do ciclo vital familiar em que os cuidadores frequentemente se encontram implica cuidar de filhos jovens e manter uma vida profissional ativa, mas os cuidados com a saúde do idoso passam para primeiro plano e esse foco tem implicações a nível familiar e profissional do cuidador (Imaginário, 2003; Franks \& Stphen, 1996).

A incapacidade do idoso influencia o ajustamento marital do seu cuidador (Andrasik \& Passchier, 2003) e está também associada à depressão no cuidador (Draper, Poulos, Cole, Poulos, \& Ehrlich, 1992). O impacto da dependência exerce repercussões a nível familiar, ao nível dos papéis e responsabilidades, bem como nos padrões de interação familiar. Rolland (1994) salienta que a estrutura e qualidade da relação do casal é influenciada pelos recursos que a família possui em lidar com a dependência dum familiar. Os cuidadores de idosos dependentes, na sua maioria, são do sexo feminino e casados (Brito, 2002), encontram-se numa relação conjugal que pode ficar comprometida, bem como as relações familiares entre pais e filhos (Imaginário, 2003).

$\mathrm{O}$ ajustamento conjugal, em vários estudos, mostrou ser uma variável moderadora na relação entre a depressão e a qualidade de vida e entre a sobrecarga e a qualidade de vida. Ashmore, Emery, Hauck e MacIntyre (2005) verificaram, em pacientes com doença pulmonar obstrutiva crônica e respectivos parceiros, que as percepções de ajustamento de casal constituíam variáveis preditoras da qualidade de vida após a reabilitação do doente. Pacientes com pobre ajustamento marital, no início do programa, experienciaram uma grande melhoria na morbidade psicológica após reabilitação. Também na doença oncológica, a literatura refere que o ajustamento de casal é um fator preditor de morbidade psicológica, i.e., fazer parte de uma díade bem ajustada serve de fator protetor (Banthia, Malcarne, Narni, Sadler, \& Greenbergs, 2004). De fato, a associação entre a qualidade da relação conjugal e a qualidade de vida tem vindo a ser demonstrada na literatura sobre relacionamento conjugal (Whisman, 2001). A discórdia marital ou a baixa satisfação com a 
relação conjugal aumenta por si só o risco de depressão, limitando ou removendo recursos como o apoio do parceiro (Proux, Helma \& Buehler, 2007). Numa situação de prestação de cuidados, torna-se assim fundamental avaliar o ajustamento conjugal. De fato, Egh, Rapport, Coleman e Hank, (2002) verificaram que o suporte social moderava o distress psicológico em cuidadores.

A sobrecarga está associada negativamente com a qualidade de vida. Brito (2002) e Garret, Martins e Ribeiro (2004) sugerem que as pessoas prestadoras de cuidados durante longos períodos, como acontece na maior parte dos casos de familiares de idosos dependentes, frequentemente sofrem alterações ao nível da qualidade de vida em diversas áreas: alterações na vida familiar e social, problemas econômicos e laborais, cansaço, e desgaste prolongado a nível psíquico e físico.

A satisfação com o apoio social é uma das variáveis associadas à satisfação com a vida e à qualidade de vida (Hohaus \& Berah, 1996). A diminuição das atividades sociais devido aos cuidados que têm que ser prestados pode fazer com que o cuidador principal se isole, ficando em situação de vulnerabilidade social (Salgueiro, 2008; Rolland, 1994; Sequeira, 2007).

Os cuidadores estão sujeitos a sentimentos de ansiedade, stress, angústia e desespero (Williamson \& Schulz, 1990). A presença de ajuda na prestação de cuidados representa um efeito protetor face ao aparecimento de sobrecarga, essencialmente ao nível da diminuição da intensidade do cuidar, favorecendo a possibilidade de desempenhar outras atividades, minorando as limitações sociais e permitindo a possibilidade de partilha e ventilação dos problemas (Brito, 2002; Lage, 2004). Assim, a presença de um cuidador secundário é um bom preditor da percepção da saúde e da qualidade de vida, bem como da sobrecarga (Lage, 2005). Os cuidadores que poderem contar com alguém sentem-se menos pressionados em relação às exigências dos cuidados, melhorando, consequentemente, a sua percepção de saúde e qualidade de vida (Brito, 2002; Lage, 2005; Williamson \& Schulz, 1990). No presente estudo, cuidador secundário foi identificado como alguém que substituía o cuidador principal nas suas tarefas quando este por motivos pessoais ou profissionais não o podia fazer, tratando-se assim, duma ajuda eventual.

$\mathrm{O}$ presente estudo pretendeu avaliar a relação entre as variáveis qualidade de vida, sobrecarga, suporte social e morbidade psicológica; as diferenças nas referidas variáveis em função do tipo de cuidador, presença versus ausência de cuidador secundário e gravidade da dependência funcional do idoso; e o efeito moderador do ajustamento conjugal na relação entre a depressão e a qualidade de vida e entre a sobrecarga e a qualidade de vida.

\section{Método}

\section{Amostra}

Participaram no estudo 94 cuidadores, $80 \%$ eram do sexo feminino cuja média de idades variava entre 40 e 70 anos. Trinta e quatro por cento referiram possuir ajuda de um cuidador secundário. $63 \%$ são cuidadores de idosos totalmente dependentes do ponto de vista funcional, $8,2 \%$ de idosos gravemente dependentes, $15 \%$ de idosos moderadamente dependentes e os restantes de idosos com dependência leve. Oitenta e seis por cento estão casados ou em união de fato. Trinta por cento dos cuidadores são cônjuges, $60 \%$ filhos e os restantes possuem outro grau de parentesco ou apenas amizade pelo cuidador. Cinquenta e um por cento dos cuidadores assumiram a prestação de cuidados há mais de 5 anos, $25 \%$ entre 3 meses e um ano, $20 \%$ há mais de um ano e os restantes há menos de 3 meses.

\section{Procedimento}

A amostra deste estudo inclui cuidadores informais inscritos num centro de saúde da Zona Norte de Portugal que têm a seu encargo idosos dependentes a nível funcional que recebem cuidados domiciliários da equipa de enfermagem.

$\mathrm{O}$ estudo foi submetido à comissão de ética da Associação Regional de Saúde onde o centro de Saúde pertencia e foi aprovado. Os dados foram colhidos nas visitas domiciliárias a todos os cuidadores, identificados pela equipa de enfermagem que aceitaram participar no estudo. Como critério de seleção, foi aplicada a Escala de Barthel e apenas foram incluídos na amostra cuidadores que tinham a seu encargo idosos com dependência funcional. Os dados foram recolhidos por uma das investigadoras 
que acompanhou a equipa de enfermagem e para quem os cuidadores eram desconhecidos. A participação foi voluntária.

\section{Instrumentos}

- Questionário Sociodemográfico (Pereira \& Carvalho, 2007). O questionário inclui vários itens que fornecem informação sobre os cuidadores ao nível da idade, sexo, estado civil, situação laboral, duração dos cuidados, prática de estilos de vida saudáveis, condição de saúde e apoios recebidos.

- Índice de Barthel,(BI) (Mahoney \& Barthel, 1965). É um instrumento de avaliação das atividades de vida diárias (AVDs) que possibilita a avaliação da capacidade funcional dos idosos, composto por 10 itens. A análise fatorial identificou três fatores ("mobilidade", "higiene" e "controle dos esfincteres") que explicam $75 \%$ da variância total. Sequeira (2007), na validação portuguesa, obteve uma boa consistência interna em todos os fatores, sendo os resultados de .96 no primeiro fator, .84 no segundo fator, .87 no terceiro fator, respectivamente, e .89 na escala global. No presente estudo, só foi utilizada a escala global e o alfa global encontrado foi de .89. Um resultado elevado indica menor dependência.

- Escala de Sobrecarga do Cuidador (ZBI) (S. H. Zarit \& J. M. Zarit, 1983; Martín, 1996; Scazufca, 2002; versão portuguesa de Sequeira, 2007) permite avaliar a sobrecarga objetiva e subjetiva do cuidador informal e inclui informações sobre a saúde, vida social, vida pessoal, situação financeira, situação emocional e tipo de relacionamento. Sequeira (2007), ao validar a escala para a população portuguesa, identificou quatro fatores: impacto da prestação de cuidados, relação interpessoal, expectativas face ao cuidador e percepção de autoeficácia, que explicam $62,1 \%$ da variância. Os alfas obtidos foram respectivamente: $.93, .83, .67 \mathrm{e}$ .80. No presente estudo, só foi utilizada a escala global e o alfa obtido, na presente amostra, foi de .87 . Um resultado elevado corresponde a uma maior percepção de sobrecarga.

WHOQOL-BREF (World Health Organization Quality of Life; Skevington, Lotfy \& O'Connell, 2004; versão portuguesa de Vaz Serra et al., 2006). Este instrumento avalia a qualidade de vida nos seus diferentes domínios (físico, psicológico, relações sociais e meio ambiente) e ao nível geral (QV Geral) que inclui dois itens não contabilizados nos restantes domínios. O domínio físico é composto por 7 itens e apresenta um alfa de .87, o domínio psicológico é composto por 6 itens e apresenta um alfa de .84 , o domínio das relações sociais é composto por 3 itens e revelou um alfa de .64 e, por último, o domínio do meio ambiente que é composto por 8 itens e apresentou um alfa de .78. Na presente amostra, a consistência interna na dimensão física foi de .85; na dimensão psicológica, de .83; na dimensão relações sociais, de .65 e na dimensão ambiental, de .69. Apesar das subescalas relações sociais e meio ambiente apresentarem alfas inferiores a .70, foram mantidas no teste de hipóteses dado que, no primeiro caso, o resultado é idêntico ao do autor original e a escala apenas apresenta 3 itens e, no segundo caso, por se encontrar muito próximo de .70 .

- Escala da Satisfação com o Suporte Social (ESSS), avalia a satisfação com o suporte social recebido, incluindo a satisfação com os amigos, satisfação com a família, intimidade e atividades sociais (Ribeiro, 1999). O fator satisfação com os amigos inclui 5 itens e apresentou um alfa de .83 , o fator satisfação com a família inclui 3 itens e um alfa de .74 , o fator intimidade inclui 4 itens e um alfa de $.74 \mathrm{e}$ o fator atividades sociais inclui 3 itens e apresentou um alfa de .64. Os alfas obtidos no presente estudo foram respectivamente os seguintes: $.72, \quad .82, .47$ e .54 . Assim, as subescalas "Intimidade" e "Atividades Sociais" não foram consideradas no presente estudo. $\mathrm{Na}$ presente amostra, o alfa da escala total foi de .80 .

- Escala de Ajustamento Conjugal (R$D A S$ ) (Spanier, 1976; versão portuguesa de Pereira, 2003). Esta escala avalia as componentes do ajustamento conjugal (Spanier, 1976; versão Portuguesa de Pereira, 2003). É constituída por 14 itens subdivididos em 3 subescalas: consenso, satisfação e coesão. De acordo com Spanier (1976), a escala total apresentou um alfa de .90 e as subescalas: .81, $.85 \mathrm{e} .80$, respectivamente. $\mathrm{O}$ alfa total no presente estudo foi de .80 . Relativamente à subescala consenso, o alfa obtido foi .80 ., e .75 na subescala satisfação e .68 na subescala coesão. Dado que o e alfa da subescala coesão se encontra muito perto de .70 e dada a sua relevância clínica, decidimos pela sua utilização no presente estudo (Nunnally, 1978). 
- Inventário de Beck para a Depressão (BDI), (Beck, Ward, Mendelsohm, Mock, \& Erbaugh, 1961; versão portuguesa de L. McIntyre e S. McIntyre, 1995a). Este questionário é composto por vinte e um itens de autoavaliação. $\mathrm{Na}$ análise fatorial realizada, derivaram-se duas subescalas. A primeira constituída por treze itens e parece referir-se a uma dimensão cognitiva da depressão e a segunda é constituída por apenas oito itens e parece referir-se a uma dimensão somática da depressão. O coeficiente alfa de Cronbach da primeira subescala é de .84 e da segunda, de .76. No presente estudo, o alfa obtido foi de .89 .

- Inventário de Avaliação da Ansiedade Estado e Traço (STAI), (Spielberger, Gorush, Lushene, Vasg, \& Jacobs, 1983; versão portuguesa de L. McIntyre \& S. McIntyre, 1995b), constituído por duas escalas de vinte itens. Na versão portuguesa, os alfas para a escala ansiedade estado e ansiedade traço foram respectivamente .89 e .88 . No presente estudo, os alfas obtidos foram .94 para a escala de ansiedade estado e de .92 para a escala ansiedade traço.

\section{Análise de dados}

Para testar a relação entre as diferentes variáveis psicológicas, foi utilizado o coeficiente de correlação de parcial de Pearson controlando a capacidade funcional do idoso.

Para avaliar as diferenças nas variáveis psicológica em função do tipo de cuidador (cônjuges, filhos e outros), foi utilizado o teste KruskWallis. Para testar as diferenças no ajustamento conjugal (total e subescalas), tendo em consideração a presença ou ausência de cuidador secundário, recorreu-se a uma Manova, dado as variáveis dependentes estarem moderadamente relacionadas.

No sentido de avaliar as diferenças entre cuidadores que cuidam de idosos total ou gravemente dependentes do ponto de vista funcional versus cuidadores moderada/levemente dependentes, foram constituídos dois grupos: no primeiro grupo, a pontuação máxima em termos do índice de Barthel vai até 35 pontos e, no segundo caso, entre 40 a 90 pontos segundo o autor. Dado que o grupo dos idosos "moderada a ligeiramente dependentes" possuía um $\mathrm{n}$ de 29 , foi utilizado o teste Man-Whitney.
Para verificar se o ajustamento conjugal funcionava como variável moderadora, recorreu-se a uma análise de regressão linear múltipla (método enter) utilizando o método proposto por Baron e Kenny (1986) nos diferentes domínios da qualidade de vida e sobrecarga.

\section{Resultados}

\section{Relação entre qualidade de vida, sobrecarga, suporte social, ajustamento conjugal e morbidade psicológica}

Ao nível da qualidade de vida, o domínio físico correlacionou-se positivamente com a satisfação com amigos $(\mathrm{r}=.296 ; \mathrm{p} \leq 05)$, satisfação com a família ( $\mathrm{r}=.255, \mathrm{p} \leq .05)$, suporte social $(\mathrm{r}=.442, \mathrm{p} \leq .001)$, ajustamento conjugal total $(\mathrm{r}=.331, \mathrm{p} \leq .001)$ e subescalas: consenso $(\mathrm{r}=.286, \mathrm{p} \leq .05)$ e satisfação marital $(\mathrm{r}=.374, \mathrm{p} \leq .001)$ e negativamente com a depressão ( $\mathrm{r}=-.506 ; \mathrm{p} \leq .001$ ), ansiedade estado $(\mathrm{r}=-.429 ; \mathrm{p} \leq .001)$ e ansiedade traço $(\mathrm{r}=-.524$; $\mathrm{p} \leq .001$ ). O mesmo aconteceu com o domínio psicológico da qualidade de vida que se correlacionou positivamente com as mesmas variáveis anteriormente citadas: satisfação com os amigos, $(\mathrm{r}=.322, \mathrm{p} \leq .001)$; suporte social $(\mathrm{r}=.446, \mathrm{p} \leq .001)$; consenso $(\mathrm{r}=.232, \mathrm{p} \leq .05)$, satisfação marital, $(\mathrm{r}=.233, \mathrm{p} \leq .05)$, ajustamento conjugal, $(\mathrm{r}=.265, \mathrm{p} \leq .05) \quad \mathrm{e}$, negativamente com a depressão $(\mathrm{r}=-.481$; $\mathrm{p} \leq .001)$, ansiedade estado ( $\mathrm{r}=-.661 ; \mathrm{p} \leq .001)$ e ansiedade traço $(\mathrm{r}=-.700, \mathrm{p} \leq .001)$, bem como os domínio ambiental e social da qualidade de vida cujos resultados foram respectivamente: satisfação com os amigos ( $\mathrm{r}=.473, \mathrm{p} \leq .001$; $\mathrm{r}=.235, \mathrm{p} \leq .05)$, satisfação com a família $(\mathrm{r}=.235, \mathrm{p} \leq .05 ; \mathrm{r}=.431, \mathrm{p} \leq .001)$, consenso $(\mathrm{r}=.266, \quad \mathrm{p} \leq .05 ; \quad \mathrm{r}=.333, \quad \mathrm{p} \leq .001)$, relacionamento conjugal $(\mathrm{r}=.258, \mathrm{p} \leq .05 ; \mathrm{r}=$ $.439, \mathrm{p} \leq .001)$, depressão $(\mathrm{r}=-.533, \mathrm{p} \leq .01 ; \mathrm{r}=-$ $.410, \mathrm{p} \leq .01)$, ansiedade estado ( $\mathrm{r}=-.476, \mathrm{p} \leq .01$; $\mathrm{r}=-.538, \mathrm{p} \leq .001)$ e ansiedade traço $(\mathrm{r}=-.600$, $\mathrm{p} \leq .001 ; \mathrm{r}=-.511, \mathrm{p} \leq .001)$. A dimensão social da qualidade de vida está ainda positivamente correlacionada com a satisfação marital ( $\mathrm{r}=$ $.369, \mathrm{p} \leq .001)$ e a coesão $(\mathrm{r}=283, \mathrm{p} \leq .05)$ e a dimensão ambiental da qualidade de vida com o suporte social $(\mathrm{r}=.285, \mathrm{p} \leq .001)$.

A sobrecarga está relacionada negativamente com a satisfação com os amigos $(\mathrm{r}=-.375, \mathrm{p} \leq .001)$, satisfação com a família ( $\mathrm{r}=$ $-.218 ; \mathrm{p} \leq .05)$ e suporte social total $(r=-.407$, 
$\mathrm{p} \leq .001)$ e positivamente com a depressão ( $\mathrm{r}=$ $.382, \mathrm{p} \leq .001)$, ansiedade estado $(\mathrm{r}=.530$, $\mathrm{p} \leq .001)$ e ansiedade traço $(\mathrm{r}=.584, \mathrm{p} \leq .001)$.

Diferenças na qualidade de vida, sobrecarga, suporte social, ajustamento conjugal e morbidade psicológica em função do tipo de cuidador

Verificaram-se diferenças ao nível da qualidade de vida $(\mathrm{p}=.001)$ e ansiedade estado $(\mathrm{p}=.046)$. Assim, os cuidadores cônjuges apresentam pior qualidade de vida $(\mathrm{M}=141.6$; $\mathrm{DP}=32$ ), seguidos dos cuidadores filhos $(\mathrm{M}=127 ; \mathrm{DP}=29.8)$ e do grupo de outros cuidadores $(\mathrm{M}=106, \mathrm{DP}=31.6)$. Em termos de morbidade psicológica, são os cuidadores cônjuges que apresentam mais ansiedade estado
$(\mathrm{M}=49.8, \mathrm{DP}=10.2)$, seguidos dos cuidadores filhos cônjuges $(\mathrm{M}=45.7 \mathrm{DP}=11.7) \mathrm{e}$, finalmente o grupo dos outros cuidadores $(\mathrm{M}=$ 39.3; $\mathrm{DP}=12.3$ ). Não se verificaram diferenças nas outras variáveis.

Diferenças na qualidade de vida, sobrecarga, suporte social, ajustamento conjugal e morbidade psicológica em função da presença/ausência de cuidador secundário

Os resultados obtidos (tabela 1) indicam diferenças ao nível do ajustamento de casal. Assim, cuidadores que possuem ajuda de um cuidador secundário manifestam maior satisfação conjugal. Não se encontraram diferenças nas outras variáveis psicológicas.

Tabela 1 - Resultados Significativos dos testes MANOVA ao nível Qualidade de Vida, Sobrecarga, Suporte Social, Ajustamento Conjugal e Morbidade Psicológica nos grupos de cuidadores de idosos dependentes em função da presença ou ausência de cuidador secundário ( $\mathbf{N = 9 4 ) ~}$

\begin{tabular}{lccc}
\hline \multicolumn{1}{c}{ Variáveis } & $\begin{array}{c}\text { Com Cuidador Secundário } \\
(\mathrm{n}=62) \\
\text { Média }(\mathrm{DP})\end{array}$ & $\begin{array}{c}\text { Sem Cuidador } \\
\text { Secundário }(\mathrm{n}=32) \\
\text { Média }(\mathrm{DP})\end{array}$ & MANOVA \\
& $23,57(4,243)$ & $22,85(4,7)$ & $\mathrm{F}$ \\
\hline Consenso (RDAS) & $16,242,86)$ & $14,73(3,336)$ & 0,534 \\
Satisfação (RDAS) & $9,96(4,62)$ & $9,66(5,237)$ & 0,077 \\
Coesão (RDAS) & $49,76(8,47)$ & $47,24(10,38)$ & 1,552 \\
Ajustamento Conjugal (RDAS-Total) & & &
\end{tabular}

Diferenças na qualidade de vida, sobrecarga, suporte social, ajustamento conjugal e morbidade psicológica em função da do grau de dependência do idoso

Não se verificaram diferenças nas variáveis psicológicas qualidade de vida, sobrecarga, suporte social, ajustamento conjugal e morbidade psicológica entre os grupos de cuidadores de idosos total a gravemente dependentes com os que apresentam dependência moderada a leve ao nível da qualidade vida $(\mathrm{p}=.684)$, sobrecarga $(\mathrm{p}=.684)$, ajustamento conjugal $(\mathrm{p}=.852)$, suporte social $(\mathrm{p}=.414)$ e morbidade psicológica: depressão: $(\mathrm{p}=.997)$, ansiedade estado ( $\mathrm{p}=.687)$ e ansiedade traço $(\mathrm{p}=.159)$.
Efeito moderador do ajustamento conjugal na relação entre a depressão e a qualidade de vida e entre a sobrecarga e a qualidade de vida

A tabela 2 revela que a interação da variável depressão com a variável moderadora ajustamento conjugal é significativa $(B=-.352$, $p \leq .001)$ qualquer que seja o nível do ajustamento conjugal total, embora a relação negativa entre a depressão e a qualidade vida seja mais intensa quando o ajustamento conjugal é elevado $(B=-.89, p \leq .001)$ do que quando o ajustamento conjugal é baixo ( $B=-$ $.34, \mathrm{p} \leq .001$ ) ( fig. 1). Desta forma, o ajustamento conjugal (total) modera a relação entre a depressão e a dimensão física da qualidade de vida. 
Tabela 2 - Ajustamento conjugal (total) como variável moderadora da relação entre depressão e a dimensão física da qualidade de vida $(\mathrm{N}=94)$

\begin{tabular}{llccc}
\hline \multicolumn{1}{c}{ Variáveis } & $\Delta \mathrm{R}^{2}$ & $\mathrm{SE}$ & Beta $(b)$ \\
\hline \multirow{2}{*}{ Bloco1 } & Depressão (BDI) & 0,309 & .880 & $-0,473^{* *}$ \\
& Ajustamento Conjugal Total (RDAS) & & .905 & 0,179 \\
& Depressão (BDI) & & .913 & $-0,63^{* *}$ \\
\multirow{2}{*}{ Bloco 2 } & Ajustamento Conjugal Total (RDAS) & 0,095 & .850 & $0,214^{*}$ \\
& Ajustamento Conjugal Total × Depressão & & .718 & $-0,352^{* *}$ \\
\hline
\end{tabular}

Nota. $\mathrm{R}^{2}$ Ajustado Bloco 1 (0.294); R ${ }^{2}$ Ajustado Bloco2 (0.384)

$* p<.05 . * * p<.001$.

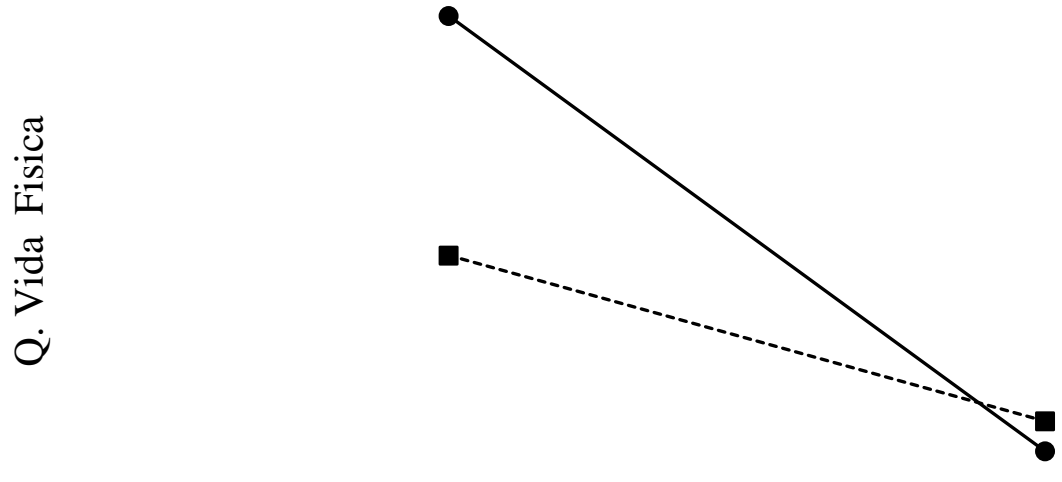

$\longrightarrow$ Ajustamento Conjugal Total

Figura 1 - Ajustamento conjugal (total) como variável moderadora na relação entre a depressão e a dimensão física da qualidade de vida

A tabela 3 revela a interação da variável depressão com a variável moderadora ajustamento conjugal é significativa $(B=-$ $0.465, \mathrm{p} \leq .001)$ qualquer que seja o nível de ajustamento conjugal, embora a relação negativa entre depressão e a dimensão psicológica da qualidade de vida seja mais intensa quando o ajustamento conjugal é elevado $(\mathrm{B}=-.98, \mathrm{p} \leq .001)$ do que quando $\mathrm{o}$ ajustamento conjugal é baixo $(B=-.30, p \leq .001)$ (fig. 2).

Verificamos que o ajustamento conjugal não modera a relação entre a depressão e a dimensão social da qualidade de vida $(B=$ 0.665, p=0.72), nem entre a depressão e a dimensão ambiental da qualidade de vida $(B=-$ $0.885, \mathrm{p}=0.85$ ). Os resultados revelaram ainda que o ajustamento conjugal também não modera a relação entre a sobrecarga e a qualidade de vida $(B=0,562, \mathrm{p}=0,92)$. 
Tabela 3 - Ajustamento conjugal total (RDAS) como variável moderadora na relação entre a depressão e a dimensão psicológica da qualidade de vida (N=94)

\begin{tabular}{llccc}
\hline \multicolumn{1}{c}{ Variáveis } & $\Delta \mathrm{R}^{2}$ & $\mathrm{~B}$ & Beta (b) \\
\hline \multirow{2}{*}{ Bloco1 } & Depressão (BDI) & 0.267 & -5.451 & $-0,465^{* *}$ \\
& Ajustamento Conjugal Total (RDAS) & & 1.483 & 0,123 \\
& & & -7.726 & $-0,66^{* *}$ \\
\multirow{2}{*}{ Bloco 2 } & Depressão (BDI) & \multirow{2}{*}{0.146} & 1.993 & 0,165 \\
& Ajustamento Conjugal Total (RDAS) & & -4.109 & $-0,437^{* *}$
\end{tabular}

Nota. $\mathrm{R}^{2}$ Ajustado Bloco1 (0.251); $\mathrm{R}^{2}$ Ajustado Bloco 2 (0.393).

$* p<.05 . * * p<.001$.

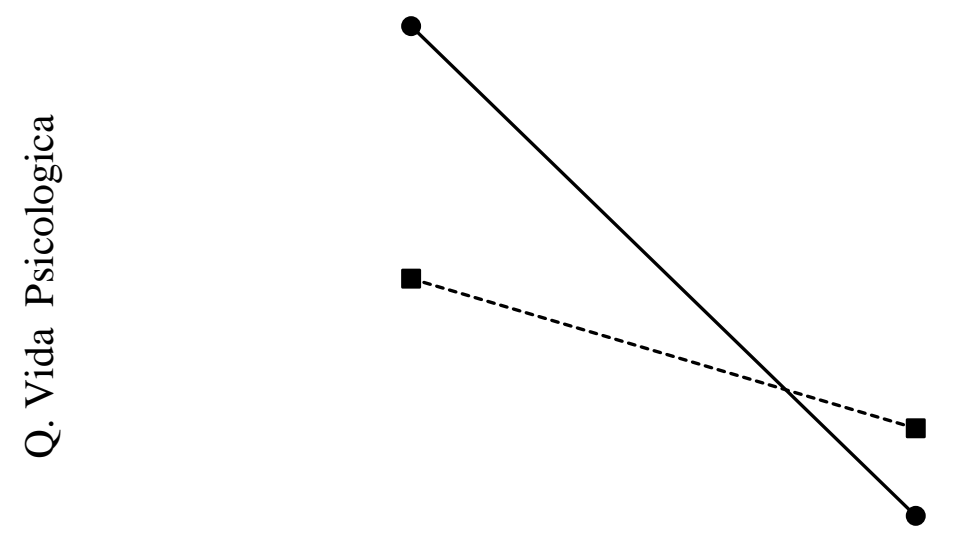

Figura 2 - Ajustamento conjugal Total (RDAS) como variável moderadora na relação entre a depressão e dimensão psicológica da qualidade de vida

\section{Discussão}

Verificou-se que, à exceção da variável coesão, todas as outras variáveis, sobrecarga, consenso, satisfação marital e morbidade psicológica, se correlacionavam com as dimensões física, psicológica e social da qualidade de vida. Maior sobrecarga e morbidade estão associadas a valores mais baixos nas dimensões física, psicológica e social da qualidade de vida. Os resultados vão de encontro a outros estudos que evidenciam uma associação fortemente significativa entre sobrecarga e sintomatologia depressiva e ansiedade, conduzindo a uma diminuição da qualidade de vida no cuidador informal (Dennis, O`Rourke, Lewis, Sharpe, \& Warlow,, 1998; Gray, 2003). A dimensão ambiental da qualidade de vida relacionou-se negativamente com a sobrecarga e com a morbidade e, positivamente com o ajustamento conjugal. Campbell, Converse e Rodger (1976) salientam que a qualidade de vida é influenciada por vários fatores, entre eles as relações conjugais e familiares. É comum em diversos estudos, a qualidade de vida estar associada a um bom 
relacionamento conjugal. Krausz (1988) refere que indivíduos bem ajustados maritalmente percepcionam melhor qualidade de vida (Hammerschmidt, Norgren, Sharlin, Souza, \& Kaslow, 2004).

O aumento da sobrecarga e morbidade psicológica (Depressão, Ansiedade Estado e Traço) estão associados a uma diminuição da dimensão ambiental da qualidade de vida. Por último, melhor satisfação com os amigos e menor morbidade psicológica estão associados a uma diminuição da sobrecarga. De fato, cuidadores com altos níveis de sobrecarga manifestam um aumento de sintomatologia depressiva, ansiedade e stress (Gray, 2003). A continuidade do desempenho do papel de cuidador pode conduzir à exaustão, podendo levar a uma situação de crise e rotura manifestando sintomas de tensão, fadiga, stress, ansiedade e frustração como consequência dos efeitos negativos da prestação dos cuidados (Zarit et al., 1980; Anderson, Linto \& StewartWynne, 1995). Neste sentido, os cuidadores apresentam uma morbidade geral superior a não cuidadores (Pinquart \& Sorensen, 2003). Por outro lado, a presença de suporte familiar e de amigos está associada a uma diminuição dos efeitos do stress no indivíduo e sistema familiar (Brito, 2002).

Verificou-se que são os cuidadores cônjuges os que apresentam pior qualidade de vida e mais ansiedade quando comparados com os cuidadores filhos adultos e outro tipo de cuidadores. Estes resultados estão de acordo com a literatura. De fato, a doença crônica de um familiar acarreta exigências na vida do casal (Gordon \& Perrone, 2004), implicando necessárias reorganizações dos papéis do casal, alterando a natureza da relação do casal (Ohman \& Soderberg, 2004). Vários estudos mostraram que a natureza da relação marital sofre o efeito de cuidar de um idoso com incapacidade funcional (Andrasik \& Passchier, 1993). Wheis, Fisher e Baird (2002) salientam que estar casado pode refletir um compromisso forte com a relação e cuidar de um terceiro elemento pode afetar negativamente a dinâmica conjugal. Assim, faz sentido que os cônjuges que partilham uma vida pré-incapacidade sejam os que mais sejam afetados quando passam a cuidadores, tendo que reorganizar a identidade e dinâmica conjugal.

Os resultados obtidos sugerem ainda que cuidadores que possuem ajuda na prestação de cuidados ao idoso dependente apresentam maior satisfação conjugal. De fato, a insegurança na prestação de cuidados, provocada pela falta de ajuda e a ansiedade associada, tem sido relacionada com uma maior insatisfação conjugal e com depressão (Kim, Carver, Deci, \& Kasser, 2008). Não foram encontrados na literatura estudos que evidenciem uma associação significativa entre a existência de um cuidador secundário e maior satisfação conjugal no cuidador principal, contudo diversos autores referem que a existência de um cuidador secundário minimiza as consequências do cuidado, traduzindo-se numa importante fonte de apoio social para os cuidadores principais (Martín, 1996; Franks \& Stphen, 1996; Martín, 2005; Heller \& Factor, 1993). O processo de cuidar de um idoso dependente é contínuo e quase sempre irreversível (Garret, Martins \& Ribeiro, 2003) pelo que a existência de alguém de retaguarda que possa substituir o cuidador temporariamente, torna-se fulcral, proporcionando ao cuidador principal maior disponibilidade para se dedicar aos restantes membros da família e a si mesmo.

Não se verificaram diferenças nas variáveis psicológicas em função da gravidade de dependência funcional do idoso. Pensamos que este resultado pode ter a ver com o fato de haver pouca variabilidade na variável. De fato, na presente amostra, a grande maioria dos idosos apresentava dependência funcional muito elevada. Assim, em futuros estudos, seria importante incluir amostras com diferentes graus de dependência funcional.

No presente estudo, verifica-se que o ajustamento conjugal é uma variável moderdora na relação entre a dimensão física e psicológica da qualidade de vida e a depressão, qualquer que seja o nível de ajustamento conjugal (total). Assim, verifica-se que cuidadores com depressão manifestam menor qualidade de vida nas suas dimensões física e psicológica em situações de elevado e baixo ajustamento conjugal. De fato, alguns estudos referem que valores elevados de depressão conduzem a uma manifestação de pior qualidade de vida (Martín, 1996; Salgueiro, 2008). Todavia, se o cuidador possuir um ajustamento conjugal elevado, mais intensa é a relação negativa entre a depressão e as dimensões física e psicológica da qualidade de vida do que quando o ajustamento conjugal é baixo. McPherson et al. (2010) referem a motivação intrínseca do cuidador como 
redutora da sua angústia psicológica, em relação à sua motivação extrínseca, incentivada pela família e sociedade. Podemos especular que, no caso de um bom ajustamento conjugal, o cuidador tem o apoio do parceiro(a) em relação à função de cuidar, podendo, desta forma, advir maior motivação intrínseca por parte do cuidador e, neste caso, a depressão poderá conduzir a um elevado stress psicológico que se repercute de forma mais evidente na qualidade de vida (Ciechanowski, Katon \& Russo, 2000).

Por outro lado, a estrutura da relação do casal pode influenciar a forma como os indivíduos lidam com a dependência, bem como percepcionam a sua saúde física, mental e emocional (Rolland, 1994; Feeney \& Hohaus, 2001), uma vez que a instalação da dependência no seio familiar tem o potencial de afetar a relação marital por alterar frequentemente a estrutura familiar (Andrasik, Larsson, \& Grazzi, 2002; Wheis et al., 2002; Wright, 2003). Assim, quando o cuidador tem um bom ajustamento conjugal, poderá ficar mais vulnerável ao impacto das alterações estruturais envolvidas com o cuidar e ao efeito negativo da depressão (stressor adicional) na sua qualidade de vida.

No que respeita à relação entre a depressão e a dimensão social da qualidade de vida e entre a depressão e a dimensão ambiental da qualidade de vida, o ajustamento conjugal não apresentou qualquer efeito moderador bem como ao nível da relação entre a sobrecarga e a qualidade de vida. De fato, é sobretudo ao nível das dimensões psicológica e física da qualidade de vida que o ajustamento conjugal se torna relevante (Rolland, 1994; Kiecolt-Glaser \& Newton, 2001). Provavelmente, ao nível das dimensões ambiental e social da qualidade de vida, variáveis como o apoio social e coping poderão desempenhar um papel mais relevante. São necessários estudos futuros para testar tais hipóteses.

Também é importante referir os aspectos culturais na relação entre os cuidadores e os familiares idosos que este estudo não incluiu. De fato, essa relação pode ser determinante ao nível da depressão e qualidade de vida do cuidador. De fato, na cultura portuguesa, existe uma expectativa da obrigatoriedade dos familiares próximos cuidarem dos seus idosos sem os institucionalizarem. Assim, é maior a probabilidade da família incluir o idoso no seu seio familiar independentemente do tipo de relacionamento existente entre ambos. Estudos futuros deveriam, assim, avaliar este tipo de relação particularmente entre cuidadores e membros familiares dependentes, pois teoricamente faz mais sentido que os cuidadores com uma má relação com o familiar de quem cuidam apresentem mais sobrecarga, depressão e menor qualidade vida já que existe maior probabilidade do familiar ser menos cooperativo e a não cooperação ser um forma do familiar dependente ter algum sentido de controle nas sua vida (Sanders, 2003).

Os resultados do efeito moderador do ajustamento conjugal reforçam a importância do ajustamento conjugal na qualidade de vida dos cuidadores (Pistrang, Barber \& Rutter 1997). Outros estudos efetuados no âmbito da doença crônica revelaram a importância da satisfação conjugal ou ajustamento conjugal (Wenger, 1990) como variável moderadora entre a morbidade psicológica e a qualidade de vida. Malheiro (2009) verificou que o ajustamento conjugal era uma variável moderadora entre a depressão e a qualidade de vida em doentes com diagnóstico de fibromialgia e Duarte (2008) verificou, em doentes com lúpus, que o ajustamento conjugal moderava a relação entre a depressão e a dimensão física da qualidade de vida.

\section{Limitações}

Este estudo apresenta algumas limitações metodológicas que devem ser consideradas na interpretação dos resultados. $\mathrm{O}$ fato da amostra ter sido exclusivamente recolhida na Zona Norte de Portugal, o uso exclusivo de medidas de autorrelato e o tamanho da mostra devem ser tidos em consideração na interpretação dos resultados. Por outro lado, o fato dos idosos apresentarem, na sua grande maioria, forte dependência funcional exige precaução na interpretação dos resultados. Assim, os dados obtidos não podem ser estendidos para a população geral, uma vez que a amostra não é representativa da população portuguesa.

Pode-se ainda considerar uma limitação do estudo, a baixa consistência interna obtida ao nível das subescalas "Intimidade" e "Atividades Sociais" da escala de satisfação do suporte social que levou a que estas não fossem consideradas no nosso estudo. Além disso, de referir que duas subescalas da qualidade de vida apresentaram alfas abaixo do 0.70 pelo 
que se sugere que este estudo seja replicado numa amostra maior.

\section{Conclusão}

Cuidadores de idosos funcionalmente dependentes com um bom ajustamento conjugal manifestaram valores mais baixos de sobrecarga e níveis mais altos de qualidade de vida. Verificou-se ainda que cuidadores de idosos dependentes com morbidade psicológica (depressão, ansiedade traço e estado) apresentaram altos níveis de sobrecarga e baixos níveis de qualidade de vida. $\mathrm{O}$ ajustamento conjugal revelou-se uma variável moderadora entre a depressão e as dimensões física e psicológica da qualidade de vida, enfatizando a importância da intervenção psicológica ter em consideração o ajustamento conjugal do cuidador, dado tratar-se duma variável moderadora com impacto na qualidade de vida do cuidador.

Tendo em conta os resultados obtidos, a intervenção domiciliária feita pelos profissionais das equipes multidisciplinares deve incluir o apoio à díada idoso/ cuidador. Os serviços comunitários devem também intervir precocemente junto dos cuidadores informais, reduzindo a tensão física e psicológica causada pela dependência, contribuindo assim, para a redução dos níveis de sobrecarga do cuidador.

Acreditamos que uma atenção redobrada ao cuidador informal terá como resultado a promoção da manutenção do idoso no domicílio, menores dificuldades nos cuidados prestados e maior satisfação com o papel de cuidador que se repercutirá também no próprio idoso.

\section{Referências}

Aleixo, S. M. P. C., Augusto, B. M. J., \& Gomes, M. Z. C. S. (2002). Cuidados Continuados: Família, Centro de Saúde e Hospital como Parceiros no Cuidar ( $1^{\mathrm{a}} \mathrm{ed}$.). Coimbra: Formasau.

Anderson, C., Linto, J., \& Stewart-Wynne, E. (1995). A population based assessment of the impact and burden of caregiving for long-term stroke survivors. Stroke, 26(5), 843- 849.
Andrasik, F., Larsson, B., Grazzi, L. (2002). Biofeedback treatment of recurrent headaches in children and adolescents. In V. Guidetti, G. Russell, M. Sillanpaa, \& P. Winner (Eds.), Headache and migraine in childhood and adolescence (pp. 317-332). London: Martin Dunitz.

Andrasik, F., \& Passchier, J. (1993). Psychological aspects. In J. Olesen, P. TfeltHansen \& K. M. A. Welch (Eds.), The Headaches (pp. 195-219). New York: Raven Press.

Ashmore, J. A. A., Emery, C. F., Hauck, E. R., \& McIntyre N. R. (2005). Marital adjustment among patients with chronic obstructive pulmonary disease who are participating in pulmonary rehabilitation. Heart \& Lung: The Journal of Acute and Critical Care, 34(4), 270-278.

Banthia, R., Malcarne, V. L., Narni, J. W., Sadler, G. R., Greenbergs, H. L. (2004). The effects of dyadic strength and coping styles on psychological distress $\mathrm{n}$ couples faced with prostate cancer. Journal of Behavioral Medicine, 26(1), 31-52.

Baron, R. M., \& Kenny, D. A. (1986). The Moderator-Mediator variable distinction in social psychological research: Conceptual, strategic, and statistical considerations. Journal of Personality and Social Psychology, 51, 1173-1182.

Beck, A. T., Ward, C. H., Mendelsohm, M., Mock, J., \& Erbaugh, J. (1961). An Inventory for measuring Depression. Archives of General Psychiatry, 4(6), 561571.

Brito, L. (2002). A saúde mental dos prestadores de cuidados a familiares idosos. S/e. Coimbra: Quarteto Editora.

Campbell, A., Converse, P. E., \& Rodger, W. L. (1976). The Quality of American Life: Perceptions, Evaluation and Satisfaction. New York: Russell Sage Foundation.

Ciechanowski, P. S, Katon, W. J., \& Russo, J. E. (2000). Depression and Diabetes: Impact of Depressive Symptoms on Adherence Function, and Costs. Archives of Internal Medicine, 160, 3278-3285. 
Dennis, M., O`Rourke, S., Lewis, S., Sharpe, M., \& Warlow, C. (1998). A quantitative study of the emotional outcome of people caring for stroke survivors. Stroke, 29, 1867-1872.

Draper, B.M., Poulos, C. J., Cole, A. M., Poulos , R. G. \& Ehrlich, F. ( 1992). A comparison of caregivers for elderly stroke and dementia victims. Journal of American Geriatric Society, 40(9), 896-901.

Duarte, S. (2008): Variáveis Individuais $e$ Familiares no Lúpus Eritematoso Sistémico. Dissertação de Mestrado em Psicologia da Saúde. Universidade do Minho.

Egh, T. C., Rapport, L. J., Coleman, R. D., \& Hanks, R. A. (2002). Predictors of caregiver and family functioning following traumatic brain injury: Social support moderates caregiver distress. Journal of Head Trauma Rehabilitation, 17,155-174

Feeney, J. A., \& Hohaus, L. (2001). Attachment and Spousal Caregiving. School of Psychology, University of Queensland and School of Applied Psychology, Grijcfith University.

Franks, M., \& Stphen, M. P. (1996). Social support in the context of caregiving: husband's provision of support to wives involved in parent care. Journal of Gerontology: Psychological Sciences, 51, 43-52.

Garret, C., Martins, T., \& Ribeiro, J. L. P. (2003). Estudo de Validação do Questionário de Avaliação da Sobrecarga para Cuidadores Informais. Psicologia, Saúde \& Doenças, 4(1), 131-148.

Garret, C., Martins, T., \& Ribeiro, J. L. P. (2004). Ansiedade e Depressão em Cuidadores Informais de Pessoas com Incapacidade Funcional. In J. Ribeiro \& I. Leal (Orgs.). Actas do $5^{\circ}$ Congresso Nacional de Psicologia da Saúde (pp. 415421). Lisboa: Fundação Calouste Gulbenkian.

Gordon, P. A., \& Perrone, K. M. (2004). When spouses become caregivers: counseling implications for younger couples. Journal of Rehabilitation, 70(2), 27-32.
Gray, L. (2003). Caregiver Depression: a growing mental health concern. Policy Brief, Family Caregiver Alliance, National Center on Caregiving, September, 1-8.

Hammerschmidt, H., Norgren, M. B. P., Sharlin, S. A., Souza, R. M., \& Kaslow, F. (2004). Satisfação conjugal em casamentos de longa duração: Uma construção possível. Estudos de Psicologia, 9(3), 575- 584.

Heller, T., \& Factor, A. R. (1993). Support systems, well-being, and placement decision-making among older parents and their adult children with developmental disabilities. In E. Sutton, A. R. Factor, B. A. Hawkins, T. Heller, \& G. B. Seltzer (Eds.), Older adults with developmental disabilites (pp. 107-122). Baltimore: Paul H. Brookes.

Hohaus, L., \& Berah, E. (1996). Stress, achievement, marriage and social support: Effects on the psychological well-being of physicians entering midlife/mid-career. Psycholgy \& Health, 11(5), 715-731.

Imaginário, C. M. I. (2003). O idoso dependente em contexto familiar. S/e. Coimbra: Formasau.

Jamura, D. (1997). Stress Dimensions among Caregivers of the Elderly. Indian Journal of Medical Research, 106, 381-388.

Kiecolt-Glasser, K. J. K., \& Newton, N. T. (2001). Marriage and Health: His and Hers. Psychological Bulletin, 127, 472- 503.

Kim, Y., Carver, S. C., Deci, E. L., \& Kasser, T. (2008). Adult attachment and psychological well- being in cancer caregivers: The Meditational Role of Spouses motives for caregiving. Health Psychology, 27, Special Issue, 144-154.

Krausz, S. (1988). Illness and loss: Helping couples cope. Clinical Social Work Journal, $16,52-65$.

Lage, M. I. (2004). Cuidar dos cuidadores de idosos dependentes. Actas do Congresso Nacional de Psicologia da Saúde. Lisboa, 5 , 749-756.

Lage, M. I. (2005). Cuidados familiares a idosos. In C. Paul, \& A. M. Fonseca. Envelhecer em Portugal. Lisboa: Climepsi Editores. 
Mahoney, F. I., \& Barthel, D. (1965). Functional evaluation: The Barthel Index. Maryland State Medical Journal, 14, 56-61.

Malheiro, M. (2009). Variáveis Psicossociais na Fibromialgia: Um Estudo de Casal. Dissertação de Mestrado em Psicologia da Saúde. Universidade do Minho.

Martín, I. (2005). O Cuidado Informal no Ámbito Social. In C. Paúl, \& A. M. Fonseca (Eds.), Envelhecer em Portugal. Lisboa: Climepsi Editores.

Martín, M. (1996). Adaptación para nuestro medio de la escala de sobrecarga del cuidador (Caregiver Burden Interview) de Zarit. Revista de Gerontologia, 6, 338-346.

Martins, R. (2004). Qualidade de vida dos idosos da região de Viseu. (Tese de doutorado não-publicada). Universidade de Extremadura, Portugal.

McIntyre L., \& McIntyre, S. (1995a). Inventário de Beck para a Depressão (BDI). Universidade do Minho, Braga, Portugal.

McIntyre L., \& McIntyre, S. (1995b). Inventário de Ansiedade Estado e Traço (STAI). Universidade do Minho, Braga, Portugal.

McPherson, C. J., Wilson, K. G., Chyurlia, L., $\&$ Leclerc, C. (2010). The balance of give and take in caregiver-partner relationships: An examination of self-perceived burden, relationship equity, and quality of life from the perspective of care recipients following stroke. Rehabilitation Psychology, 55(2), 194-203.

Mendes, M. (2004). Mudanças Familiares ao Ritmo da Doença. As implicações da doença crónica ao nível da família e do Centro de Saúde. Dissertação de Mestrado em Sociologia, Instituto de Ciências Sociais, Universidade do Minho, Braga, Portugal.

Nunnally, J. C. (1978). Psychometric theory $\left(2^{\mathrm{a}}\right.$ ed.). New York: McGraw-Hill.

Ohman, M., \& Soderberg, S. (2004). The experiences of close relatives living with a person with serious chronic illness. Qualitative Health Research, 14(3), 396410.
Parks, S, H., \& Pilisuk, M. (1991). Caregivers burden and psychological cost of caregiving. American Journal of Orthopsyquiatry, 61(4), 501-509.

Paúl, M. C. A (2005). Construção de um Modelo de Envelhecimento Humano. In C. Paúl, \& A. M. Fonseca (Coords.). Envelhecer em Portugal ( $1^{\text {a }}$ ed.). Lisboa: Climepsi Editores.

Pearlin, L., Mullan, L. T., Semple, S., \& Skaff, M. M. (1990). Caregiving and the stress process: an overview of Concepts and Their Measures. The Gerontologist, 30, 583-594.

Pereira, M. G. (2003). Revised Dyadic Adjustment Scale - Versão de Investigação. Universidade do Minho, Braga, Portugal.

Pereira, M. G., \& Carvalho, H. (2007). Questionário Sóciodemográfico para idosos - Versão de Investigação. Universidade do Minho, Braga, Portugal.

Pinquart, M., \& Sorensen, S. (2003). Differences between caregivers and noncaregivers in psychological health and physical health: A meta-analysis. Psychology and Aging, 18(2), 250-267.

Pinquart, M., \& Sorensen, S. (2011). Spouses, adult children and children-in-law as caregivers of older adult. Psychology and Aging, 26(1), 3-14.

Pistrang, N., Barber, C. \& Rutter, C. (1997). Social support as conversation: analyzing breast cancer patient's interactions with their partners. Social Science and Medicine, $45,773-782$.

Proux, C. M., Helma, C. M., \& Buehler, C. (2007 ). Marital Quality and Personal WellBeing: A Meta-Analysis. Journal of Marriage and Family, 69, 576-593.

Ribeiro, J. L. P. (1999). Escala de satisfação com o Suporte Social (ESSS). Análise Psicológica, 3(17), 547- 558.

Richards, B. S., \& Lilly, M. L. (2001). Gerontological Family Nursing. In S. M. Hanson (Ed.). Family Hearth Care Nursing. Theory, Practice and Research. Filadélfia, FA: Davies Publishers. 
Rolland, J. S. (1994). In sickness and in health: The impact in couple's relationships. Journal of Marital and Family Therapy, 20, 327-348.

Salgueiro, H. D. A. G. (2008). Percepção do Estado de Saúde e de Qualidade de Vida da Família que Coabita e Cuida de um Idoso Dependente. Revista Investigação em Enfermagem, 17, 51-61.

Sanders, G. (2003). Family relations and caregiving: The journey through caregiving. North Dakota Family Caregiver Project. North Dakota Sate University.

Scazufca, M. (2002). Versão Brasileira da Escala "Burden Interview" para avaliação de sobrecarga em cuidadores de indivíduos com doenças mentais. Revista Brasileira de Psiquiatria, 24(1), 12-17.

Sequeira, C. (2007). Cuidar de Idosos Dependentes: Diagnósticos e Intervenções. $\left(1^{\mathrm{a}}\right.$ ed.). Coimbra: Quarteto Editores.

Skevington, S. M., Lotfy, M., \& O’Connell, K. A. (2004). The World Health Organization's WHOQOL-BREF quality of life assessment: Psychometric properties and results of the international field trial. A Report from the WHOQOL Group. Quality of Life Research, 13, 299-310.

Spanier, G. B. (1976). Measuring Dyadic Adjustment: New scales for assessing the quality of marriage and similar dyads. Journal of Marriage and the Family, 38(1), 15-28.

Spielberger, C. D, Gorush, R., Lushene, R. Vasg, P. R, \& Jacobs, G. A. (1983). Manual for the State-Trait Anxiety Inventory. Palo Alto: Consulting Psychologists Press.

Vaz Serra, A., Canavarro, M. C., Simões, M. R., Pereira, M., Gameiro, S., Quartilho, M. J., et al. (2006). Estudos Psicométricos do Instrumento de Avaliação da Qualidade de Vida da Organização Mundial da Saúde (WHOQOL-Bref) para Português de Portugal. Psiquiatria Clínica, 27(1), 41-49.
Wenger, G. (1990). The special role of friends and neighbors. Journal of Aging Studies, 4(2), 149-169.

Wheis, K., Fisher, L., \& Baird, M. (2002). Families, health, and behavior. Families Systems Health, 20, 7-46.

Williamson, G. M., \& Schulz, R. (1990). Relationship orientation, quality of prior relationship, and distress among caregivers of Alzheimer`s patients. Psychology and Aging, 5, 502-509.

Whisman, M. A. (2001). The association between depression and marital dissatisfaction. In S. R. H. Beach (Ed.), Marital and family processes in depression: A scientific foundation for clinical practice (pp. 3-24). Washington, DC: American Psychological Association.

Wolff, J. L., \& Kasper, J. D. (2006). Caregivers of Frail Elders: Updating a National Profile. The Gerontologist, 46(3), 344-356

Wright, N. (2003). Moving beyond and dual diagnosis in general practice: Many patients have multiple morbidites, and their needs have to be addressed. British Medical Journal, 8, 512-514.

Zarit, S. H., Reever, K. E., \& Bach- Peterson, J. (1980). Relatives of impaired elderly: correlates of feeling of burden. The Gerontologist, 20(6), 649-655.

Zarit, S. H., \& Zarit, J. M. (1983). The Memory and Behavior Problems Checklist and the Burden Interview. Technical Repport, Pennsylvania State University.

Zhan, L. (2004). Caring for family members with Alzheimer's disease: perspectives from Chinese American caregivers. Journal of Gerontological Nursing, 30, 19-29.

Recebido em 22 de Janeiro de 2012 Texto reformulado em 18 de Abril de 2012 Aceite em 23 de Maio de 2012 Publicado em 31 de Dezembro de 2012 